\title{
Electronic Health Record Innovations for Healthier Patients and Happier Doctors
}

\author{
Alex H. Krist, MD, MPH
}

This special issue explores a range of health information technology (HIT) issues that can help primary care practices and patients. Findings address the design of HIT systems, primarily electronic health records (EHRs), the utility of various functionalities, and implementation strategies that ensure the greatest value. The articles also remind us that, while HIT can support the delivery of care, it is not a panacea. To be effective, functionality needs to be relevant and timely for both the clinician and patient. Prompts and better documentation can improve care, and "prompt fatigue" is not inevitable. Information presented within EHRs needs to be actionable. There is an ongoing tension between information overload and the right—and helpful—information. Even the order of presentation of information can make a difference in the outcome. Whether supported by HIT or not, basic tenants of care, such as including the whole care team in trainings, communicating with other providers, and engaging patients, remain essential. The studies in this issue will prove useful for informatics developers, practices and health systems making HIT decisions, and care teams refining HIT to support the needs of their patients.(J Am Board Fam Med 2015;28:299-302.)

If your practice is like mine, you cannot walk down the hall without hearing someone complain about your electronic health record (EHR). Why is the system so slow today? Why can't the EHR help me with ... [insert some important patient care task]? Why does the EHR always make me ... [insert some annoying meaningful use requirement]? Why does it take so long to ... [insert something you do dozens of times each day]?

These complaints are not surprising. An EHR is literally right in front of most clinicians' face for over 8 hours every day. Clinicians' lives revolve around their EHR. A clinician commonly accesses, views, and enters information in the EHR throughout a patient encounter. Minutes between patient time and even personal time is spent completing tasks and messaging patients through the EHR. Despite its prominence in our life, EHRs, and health information technology (HIT) more broadly, are still in their infancy.

From the Department of Family Medicine and Population Health, Virginia Commonwealth University, Richmond.

Funding: none.

Conflict of interest: none declared.

Corresponding author: Alex H Krist MD, MPH, PO Box 980251, Richmond, VA 23298-0251 (E-mail: ahkrist@vcu. edu, 804-828-9626).
We only started incenting practices and hospitals to adopt EHRs in 2009. Not until 2016 will meaningful use focus on improved outcomes. ${ }^{1}$ At best, clinicians are ambivalent as to whether they are satisfied with their EHR and whether they think it helps patients. ${ }^{2}$ In fact, clinician dissatisfaction and burnout is at an all-time high, in part directly because of the burdens imposed by EHRs. ${ }^{3}$

Yet, if your practice is like mine, you would never go back to paper records. EHRs can better organize information, remind us about what we need to do, coordinate and track activities over time and across team members, and support communication with patients and specialists. Much of what family physicians do centers on information: to make good decisions, to coordinate care, and to empower patients with information. And there is hope for the future-EHRs can get better. The secretary of Health and Human Services has gone so far as to pledge to make clinicians happier by making their EHR better. ${ }^{4}$

\section{The Future of Electronic Documentation}

Early EHRs functioned mainly as a digital version of the Article chart and a tool to support charge capture. Even today, clinical encounter notes are designed to document content needed for reim- 
bursement in a fee-for-service market. Many have criticized the quality of encounter notes generated by EHRs, stating notes are cluttered, redundant, irrelevant, duplicative, and at times spurious. ${ }^{5}$ Koopman et $\mathrm{al}^{6}$ used cognitive task analysis to simulate how clinicians prepare for an office visit to understand what information is needed from encounter notes. Clinicians identified narrative sections as having particular value, whereas check box sections and automatically cited content already available on summary dashboards had no value. The findings highlight a need to reengineer antiquated progress notes. Unlike prior paper-based notes that needed to summarize everything about a patient, electronic progress notes should be viewed as one data element in the patient's broader electronic record. This may reduce cognitive load, errors, and fatigue, as well as allow clinicians to focus more on the patient.

\section{Prompts at the Point of Care}

Four studies in this issue report the value and use of prompts at the point of care, a task particularly well suited to EHRs. Alerts and reminders can be triggered by clinical information already within or being entered into an EHR. Such prompts before and during busy encounters can be helpful in reminding about needed care. They can also be used as standing orders to better engage multiple team members to deliver care. ${ }^{7}$ Conversely, prompts can be a distraction during crowded encounters, when more pressing issues are being addressed than those prompted. This can result in alert fatigue, culminating in clinicians ignoring all prompts, even potentially useful ones. ${ }^{8}$ The studies in this issue help to define the scenarios in which prompts are useful, show us how cognitive science can create more useful prompts, and demonstrate how unique information contained within prompts can change practice patterns.

For example, Ruffin et $\mathrm{al}^{9}$ compared patient human papillomavirus vaccination rates in practices with and without a prompt and reminder system. Human papillomavirus vaccination was more likely to be initiated in the prompted cohort. More important, the prompts seemed to work better in groups at greater risk for not being vaccinated: African Americans, older patients whom clinicians might not think about vaccinating, and patients seen in the office less frequently. Zazove et $\mathrm{al}^{10}$ had a different experience. In the same group of practices with the same institution-created EHR and reminder system, they observed no change in documentation or screening interventions after instituting a simple family history prompt that identified patients at high risk for 6 common conditions. Whether the family history prompts lacked the immediacy or clear response action of the vaccination prompts or whether other factors result in differential uptake is unclear and worthy of future research.

Bunt et al, ${ }^{11}$ through a double-blind simulation, demonstrated that sharing not only guideline recommendations for the workup of pediatric hematuria but also information about radiation exposure influenced clinician decisions to lower pediatric radiation exposure. Interestingly, the order in which information was shared (guideline vs radiation exposure information) influenced outcomes. This study highlights the value of thinking outside of the box about what-and when-information should be shared in prompts to change clinician practice patterns.

Green et $\mathrm{al}^{8}$ show that prompts and alerts do not necessarily result in reminder fatigue. Clinicians in 5 practices received nearly a million reminders related to half a million encounters. Amazingly, clinicians took action to respond to $>60 \%$ of prompts and documented discussions for another $26.8 \%$. Green et al attribute this success to the use of cognitive science and human factors engineering in designing the prompts. Strategies included ensuring reminders occurred only when services were due and were actionable, allowing clinicians to choose when to attend to reminders, making reminders simple, and ensuring practices desired the reminders before they were implemented.

\section{It Takes a Team}

Through the study of meaningful use exemplars within a national network, Ornstein et al ${ }^{12}$ remind us that it takes a team to effectively use HIT. Higher-quality performance was associated with staff education, use of standing orders, and care team reminders. EHR use was necessary but not sufficient for high performance, and staff reported a greater impact when it was clear how to apply education directly to performance.

Shultz and Holmstrom ${ }^{13}$ expand our idea of who we might consider as part of our care team: scribes. 
A systematic review identified 3 high-quality studies of the impact of scribes to help clinicians cope with the difficulties, time, cost, and inefficiencies introduced by EHRs. While scribes were studied in only the emergency department and specialty offices, they improved clinician satisfaction, productivity, and the patient-clinician relationship.

The patient will always be the most essential member of any care team. Engaged, activated patients have better outcomes. Kuhn et $\mathrm{al}^{14}$ show us how HIT can be used to create an electronic asthma action plan (eAAP). While action plans are considered best practice, clinicians are not very good at routinely creating them with patients. Kuhn et al describe how a multidisciplinary team of investigators, clinicians, and health system leaders worked together to create, implement, and broadly disseminate an eAAP using their health system's EHR. The eAAP engaged $>5000$ patients to better self-manage their asthma, resulting in fewer pediatric asthma exacerbations and fewer prescriptions for oral steroids.

\section{EHRs Can Bring People Together}

At its core, primary care is about relationships. EHRs will never replace relationships, but they can facilitate communication. In a mixed methods study Liddy et $\mathrm{al}^{15}$ report clinician satisfaction for a web-based e-consult service designed to improve primary care clinician and patient access to specialist consultation. Primary care clinicians reported high satisfaction with the use of this virtual platform, citing benefits like quick response time, the helpfulness of responses, and reassurance for the patient and clinician. In previous studies the use of the virtual platform even reduced unnecessary faceto-face referrals by $40 \%$. Findings from O'Malley et $\mathrm{al}^{16}$ further reinforce the value of communication between primary care and specialists. By linking responses to a national survey with Medicare claims data, they identified that greater primary care and specialist communication is associated with reduced hospitalizations for ambulatory caresensitive conditions. This was magnified by EHR use, suggesting that EHRs can help to coordinate communication between primary care and specialty clinicians.

\section{Conclusion}

End users-clinicians and patients-need to lead the way in the rational design, implementation, and evaluation of EHRs. However, doing this is difficult. End users, who stand the most to gain from EHR use, have limited ability to make substantive changes to their EHR. The North American Primary Care Research Group's HIT committee has issued several calls to action for more primary care researchers to systematically organize these efforts through research. ${ }^{17,18}$ The authors in this HIT theme issue have answered this call and should be both listened to and commended for their pioneering studies. As the field of HIT continues to mature, more opportunities to truly modify, adapt, and test systems will present. Primary care should lead the charge to reach our goal of better HIT use to improve our patients' health outcomes.

\section{References}

1. HealthIT.gov. EHR incentives \& certification. Meaningful use definitions \& objectives. Updated February 6, 2015. Available from: http://www.healthit.gov/ providers-professionals/meaningful-use-definitionobjectives. Accessed March 19, 2015.

2. Kane L, Chesanow N. Medscape EHR report 2014. Physicians rate top EHRs. July 15, 2014. Available from: http://www.medscape.com/features/slideshow/ public/ehr2014. Accessed March 19, 2015.

3. Friedberg MW, Chen PG, Van Busum KR, et al. Research report. Factors affecting physician professional satisfaction and their implications for patient care, health system, and health policy. 2013. Available from: http://www.rand.org/content/ dam/rand/pubs/research_reports/RR400/RR439/ RAND_RR439.pdf. Accessed March 19, 2015.

4. American Medical Association: National Advocacy Conference. February 24, 2015. Available from: http:// www.hhs.gov/secretary/about/speeches/sp20150224. html. Accessed March 31, 2015.

5. Hirschtick RE. A piece of my mind. Copy-and-paste. JAMA 2006;295:2335-6.

6. Koopman RJ, Barker-Steege LM, Moore JL, et al. Physician information needs and electronic health records (EHRs): time to reengineer the clinic note. J Am Board Fam Med 2015;28:316-23.

7. Donahue K, Plescia M, Stafford K. Clinical inquiries. Do standing orders help with chronic disease care and health maintenance in ambulatory practice? J Fam Pract 2010;59:226-7.

8. Green LA, Nease RF Jr, Klinkman MS. Clinical reminders designed and implemented using cognitive and organizational science principles decrease reminder fatigue. J Am Board Fam Med 2015;28: 351-9.

9. Ruffin MT, Plegue MA, Rockwell PG, Young AP, Patel DA, Yeazel MW. Impact of an electronic health record (EHR) reminder on human papilloma- 
virus (HPV) vaccine initiation and timely completion. J Am Board Fam Med 2015;28:324-33.

10. Zazove P, Plegue MA, Uhlmann WR, Ruffin MT. Prompting primary care providers about increased patient risk as a result of family history: does it work? J Am Board Fam Med 2015;28:334-42.

11. Bunt CW, Burke HB, Towbin AJ, et al. Point-ofcare estimated radiation exposure and imaging guidelines can reduce pediatric radiation burden. J Am Board Fam Med 2015;28:343-50.

12. Ornstein SM, Nemeth LS, Nietert PJ, Jenkins RG, Wessell AM, Litvin CB. Learning from primary care meaningful use exemplars. J Am Board Fam Med 2015;28:360-70.

13. Shultz CG, Holmstrom HL. The use of medical scribes in health care settings: a systematic review and future directions. J Am Board Fam Med 2015; 28:371-81.

14. Kuhn L, Reeves K, Taylor Y, et al. Planning for action: the impact of an asthma action plan decision support tool integrated into an electronic health record (EHR) at a large health care system. J Am Board Fam Med 2015;28:382-93.

15. Liddy C, Afkham A, Drosinis P, Joschko J, Keely E. Impact of and satisfaction with a new eConsult service: a mixed methods study of primary care providers. J Am Board Fam Med 2015;28:394-403.

16. O'Malley AS, Reschovsky JD, Saiontz-Martinez C. Interspecialty communication supported by health information technology associated with lower hospitalization rates for ambulatory care-sensitive conditions. J Am Board Fam Med 2015;28:404-17.

17. Krist AH, Beasley JW, Crosson JC, et al. Electronic health record functionality needed to better support primary care. J Am Med Inform Assoc 2014;21:764-71.

18. Krist AH, Green L, Phillips RL, et al. Health information technology needs help from primary care researchers. J Am Board Fam Med 2015;28:306-10. 